Article

\title{
Do Exostoses Correlate with Contact Disfunctions? A Case Study of a Maxillary Exostosis
}

\author{
Maria Cristina Valigi ${ }^{1, *(1)}$ and Silvia Logozzo ${ }^{1,2}$ (D) \\ 1 Department of Engineering, University of Perugia, Via G. Duranti 1, 06125 Perugia, Italy; \\ sililog@hotmail.com \\ 2 Department of Research and Development, V-GER SRL, Via Bentivogli 4, 40055 Castenaso, Bologna, Italy \\ * Correspondence: mariacristina.valigi@unipg.it
}

Received: 18 January 2019; Accepted: 9 February 2019; Published: 13 February 2019

\begin{abstract}
A maxillary exostosis is a benign overgrowth of bone that occurs on the outer or facial surface of the maxilla and is usually located near the premolar or molar teeth. This paper investigates the correlation between the presence and growth of jaw exostoses and the oral mechanics of contact. For this purpose, a case study of an upper jawbone exostosis of a female patient was considered. 3D models of the patient's cranial bones were extracted from 2D computerized tomography (CT) data and were analyzed by proper software. A contact congruence evaluation based on the Winkler contact model was performed, and results were presented in terms of indentation maps and load distributions. Results were correlated with the theory of bone remodelling by Wolff.
\end{abstract}

Keywords: maxillary exostosis; bone remodelling; congruence measure

\section{Introduction}

Oral exostoses are benign bone neoformations composed entirely of compact bone, with deeper parts that may contain spongy bone. They originate from the cortical surface of the maxilla or the mandible. Oral exostoses have been extensively studied for more than one century, but they remain an unexplained anatomical trait. Historically, the onset of oral exostoses has been correlated with many aetiologies - mainly genetic, environmental, and mechanical etiologies [1-8].

Hence, the etiology of oral exostoses can be considered a complex, multifactorial process. The process includes factors related to biomechanics and mechanics of contact, according to the functional matrix hypothesis (FMH) by Moss [9-12] and the law of bone remodelling by Wolff [13].

According to the FMH, any development, growth, and change of any skeletal unit, even in terms of shape, size, and position, arises as a compensatory response to functional requirements (e.g., transmission of a mechanical load). Accordingly, the law of bone remodelling states that bone deformities are the consequence of a functional adjustment to an alteration of the biomechanical loads. From this point of view, bone deformation is not necessarily a pathologic condition, but one that is a result of mechanical or contact disfunction.

Singh uses the SMH (Spatial Matrix Hypothesis) to explain the aetiology of oral exostoses, and suggests that the maxillary bones undergo deformation processes if they are subjected to parafunctional activities, e.g., bruxism [14]. According to these theories and the law of bone remodelling, oral exostoses can grow as neoformations in response to altered mechanical loads and contacts [15]. The orientation and location of an exostosis is strongly influenced by stress direction and distribution.

In this paper, the correlation between the growth of a buccal maxillary exostosis in a female patient and mechanical contact disfunctions of the temporomandibular joint (TMJ) is investigated, with no reference to genetic or environmental issues. 


\section{Material and Methods}

\subsection{Joint Congruence and Contact Model}

In this paper the investigation of contact disfunctions was performed by considering the joint congruence theory, based on the elastic foundation contact model (EFCM) by Winkler and on the clever intuition of Conconi et al. [16-18].

The elastic foundation contact model by Winkler is also applied in civil and industrial settings [19-24], and in analyzing the contact for soft biological tissues [25].

According to Conconi et al. [16-18], joint congruence measurements can be obtained by analysing the geometrical relationships of the contact surfaces of the joint. When combined with the EFCM, congruence measurements provide information about the ratio between the applied resultant load on the joint and the peak pressure.

Congruence measurements also provide information about the ability of the joint members to distribute an applied load during contact.

Considering Figure $1, V_{1}$ and $V_{2}$ are the volumes of the two members of the joint; $S_{1}$ and $S_{2}$ are the lateral surfaces of the two members. Considering a global offset of $S_{1}$ equal to $\Delta$, the volume $V$ is generated by a Boolean intersection.

The congruence measure (CM) index, according to the Winkler EFCM model, can be obtained from:

$$
\mathrm{CM}=\frac{\mathrm{F}}{\mathrm{p}_{0}}=\frac{\mathrm{V}}{\Delta},
$$

where $\mathrm{F}$ is the total resultant force applied to the joint, $\mathrm{p}_{0}$ is the peak pressure, and $\Delta$ is the maximum offset value of the volume of one joint bone, indenting the other joint bone, corresponding to the peak value of pressure $\mathrm{p}_{0}$.

Therefore, by means of the relationship between the loads and the geometrical characteristics of the joint, it is possible to characterize the load distribution on joint surfaces based on the indentation maps.

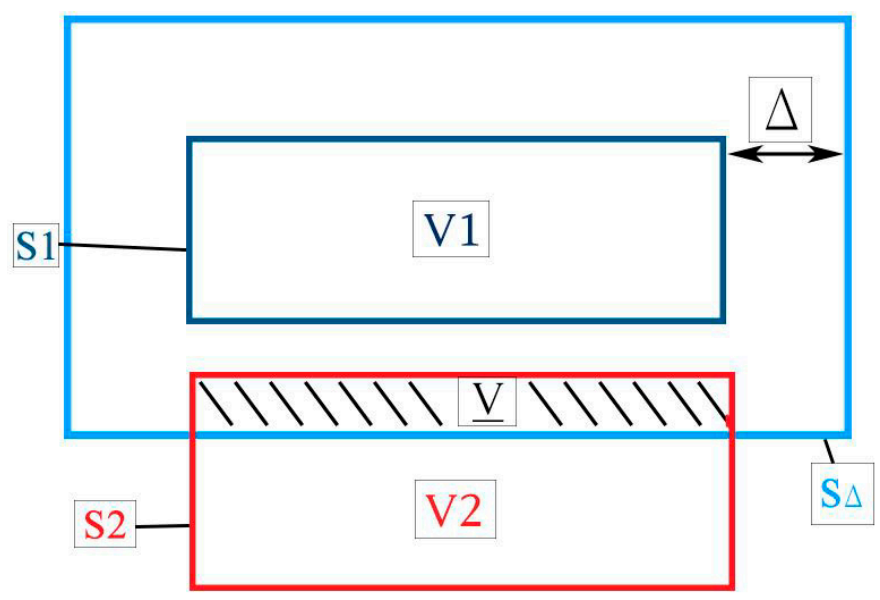

Figure 1. Cross-section of the joint and congruence geometrical parameters.

In this paper, through elaboration and theoretical evaluations based on Wolff's theory of bone remodeling, the congruence of the contact congruence was assessed and interpreted by means of indentation maps and the Winkler EFCM model.

\subsection{Case Study of a Maxillary Exostosis: Congruence and Contact Evaluation}

The case study analysed in this paper was a right buccal maxillary exostosis in a 46-year-old female patient. After obtaining informed consent, the two-dimensional images from the cranial CT (computerized tomography) of the patient (Figure 2) were further processed, in order to evaluate eventual correlations between the maxillary bone's growth and mechanical contact disfunctions. 
The CT images excluded the presence of tumoral masses and malocclusion problems, since it has been hypothesized that the presence of the exostosis could be a consequence of an abnormal or nonuniform load distribution.

The CT scan was performed using the clinical CT scanner Optima CT660 (GE Healthcare, Chicago, IL, USA). A total of 1088 images with a slice thickness between 0.625 and $2 \mathrm{~mm}$ were taken in the sagittal, frontal, and transverse planes.

The 2D CT scans were treated by using the software InVesalius 3.1.1 (Center for Information Technology Renato Archer CTI, Campinas, Brazil), an open source software for CT and MRI (magnetic resonance image) image processing. The 3D model of the entire patient's cranium was reconstructed in the form of a 3D triangular mesh (STL file) by interpolating all the slices.

This 3D model was used as the basis for the contact analysis, which was performed by applying the joint congruence theory [16-18], and comparing the healthy (left) and abnormal (right) sides of the maxilla.
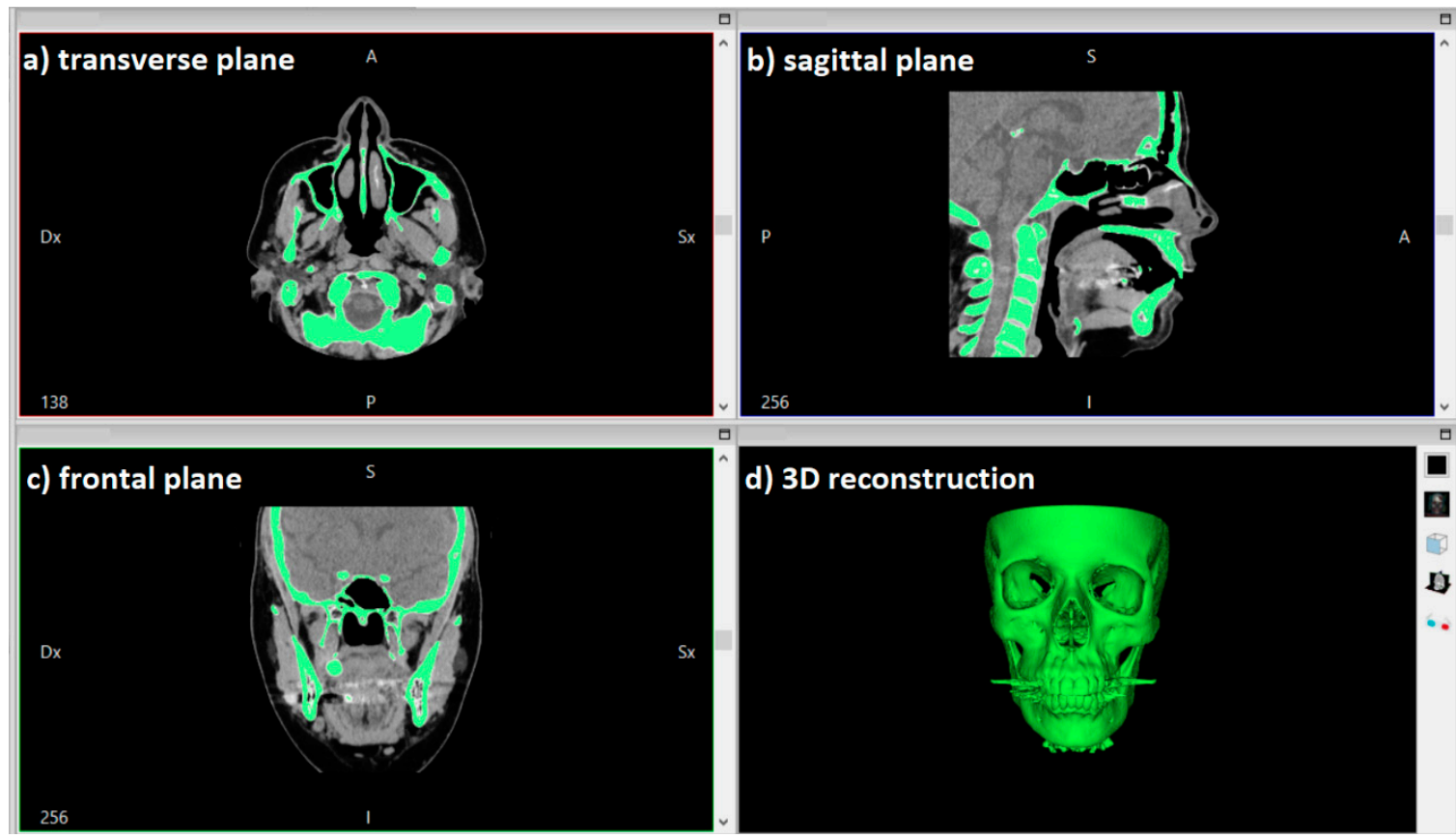

Figure 2. Cranial CT (computerized tomography) images in the anatomical planes (a-c) and 3D reconstruction $(\mathbf{d})$.

The STL (STereo Lithography interface format) 3D model was imported in the mesh editing software Meshmixer 11.3.15 (Autodesk, Inc., San Rafael, CA, USA) and Geomagic Wrap 2015.3.1 (3D Systems, Inc., Rock Hill, SC, USA).

The 3D model was optimized by reducing the noise, clusters, and self-intersections, and preserving the original shape of the bones. Then, the maxilla and mandibula were extracted and isolated by removing the teeth and filling all the holes. Further analyses of the right buccal maxillary exostosis were then conducted (Figure 3).

Removal of the teeth was consistent because the occlusion was considered optimal by the CT images and by a specific occlusion test performed during the clinical treatment of the patient.

The 3D models of the maxilla and the mandibula were left in their original position, and then joint members were treated, according to the model represented in Figure 1, in order to analyze their ability to distribute the contact loads in various geometrical configurations. 


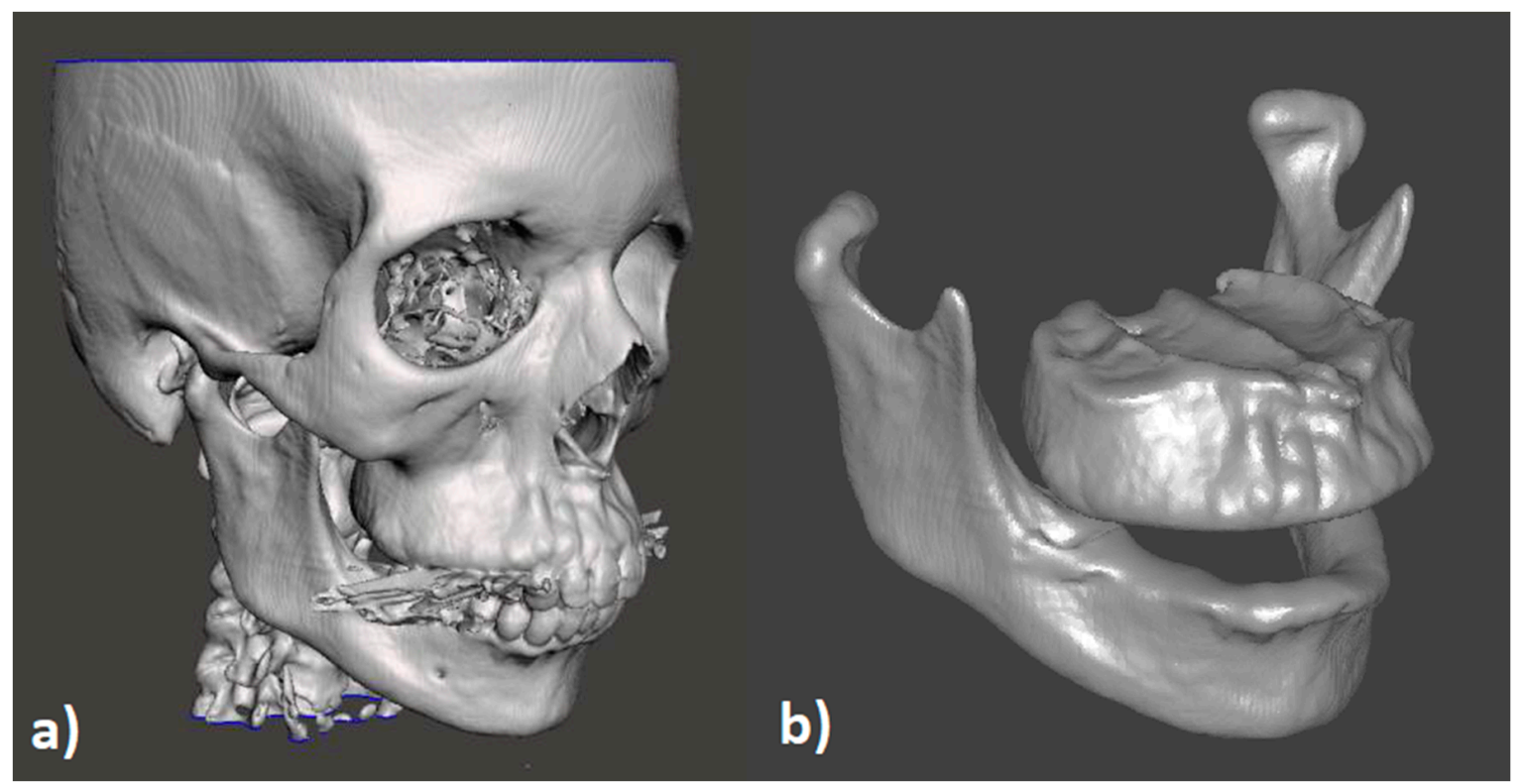

Figure 3. 3D model: (a) complete model; (b) maxilla and mandibula.

Figure 4 shows the sagittal representation of the Winkler's springs associated with the maxilla and mandibula. They were aligned almost parallel to each other and perpendicular to the articular surface, in analogy with the EFCM. This hypothesis was in agreement with the behavior of the trabecular bone supporting the joint surfaces, where trabeculae were almost normal to the contact surfaces and mainly functioned as springs in tension and compression [17].

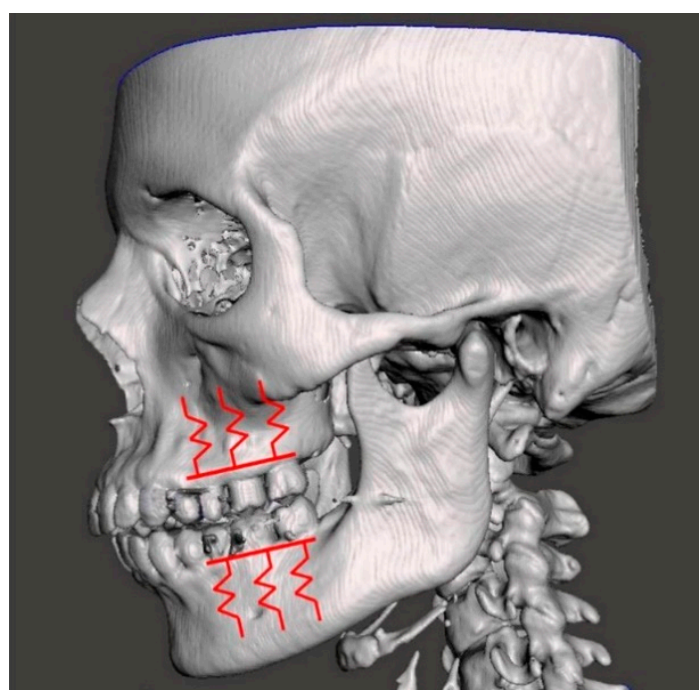

Figure 4. Elastic foundation contact model (EFCM) model of the jawbones.

According to this model, the lateral surfaces of the maxilla were offset by a value $\Delta=8 \mathrm{~mm}$. Then, the maxilla was shifted by $10 \mathrm{~mm}$ in the vertical direction to indent the volume of the mandibula (Figure 5). Thus, the 3D map of indentation was built by means of Boolean operations to evaluate the load distribution and congruence of the contact on the right and left side of the jaw bones. 


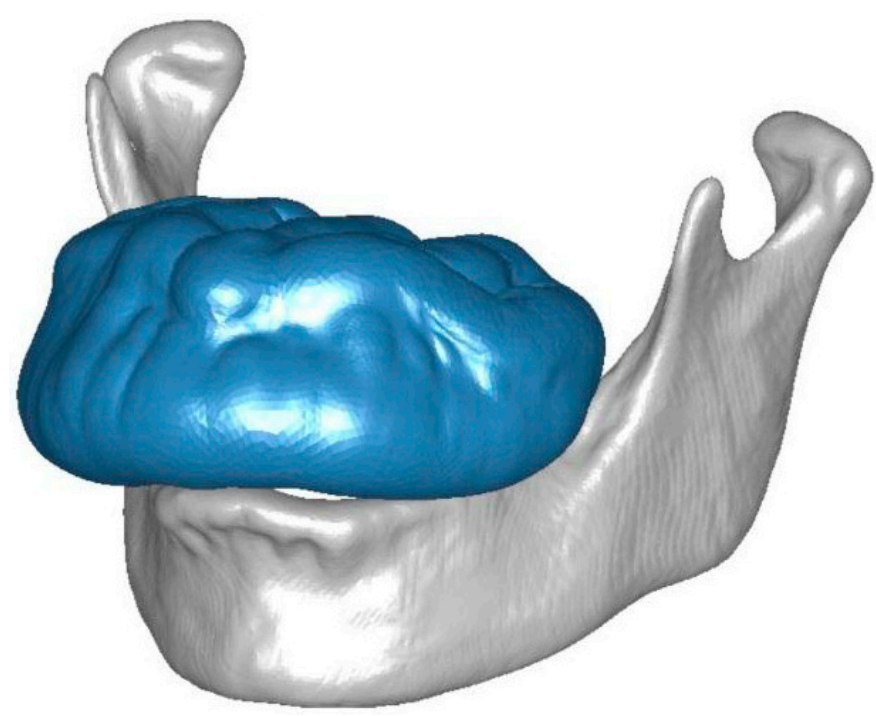

Figure 5. Maxilla geometrical treatment: offset and vertical shift.

\section{Results and Discussion}

The results were presented in terms of indentation maps, which represent the distribution of contact loads from a purely geometric analysis. The discussion dealt with assumptions that the exostosis was the result of bone remodeling, resulting from a non-uniform distribution of contact forces on the left and right side of the jaw. In particular, the indentation map representing the contact between maxilla and mandibula is represented in Figure 6. In this picture, the penetration of the offset and translated surface of the maxilla inside the mandibula is shown; the red regions indicate the surfaces where the indentation is absent, while the green and blue areas represent a deeper and deeper indentation. As mentioned above, this geometrical evidence also gives information about the distribution of the loads on the two sides of the jaw bones. In particular, one can observe that the load was higher on the right side of the jawbone, which presets the buccal exostosis, while it was lower on the opposite side.

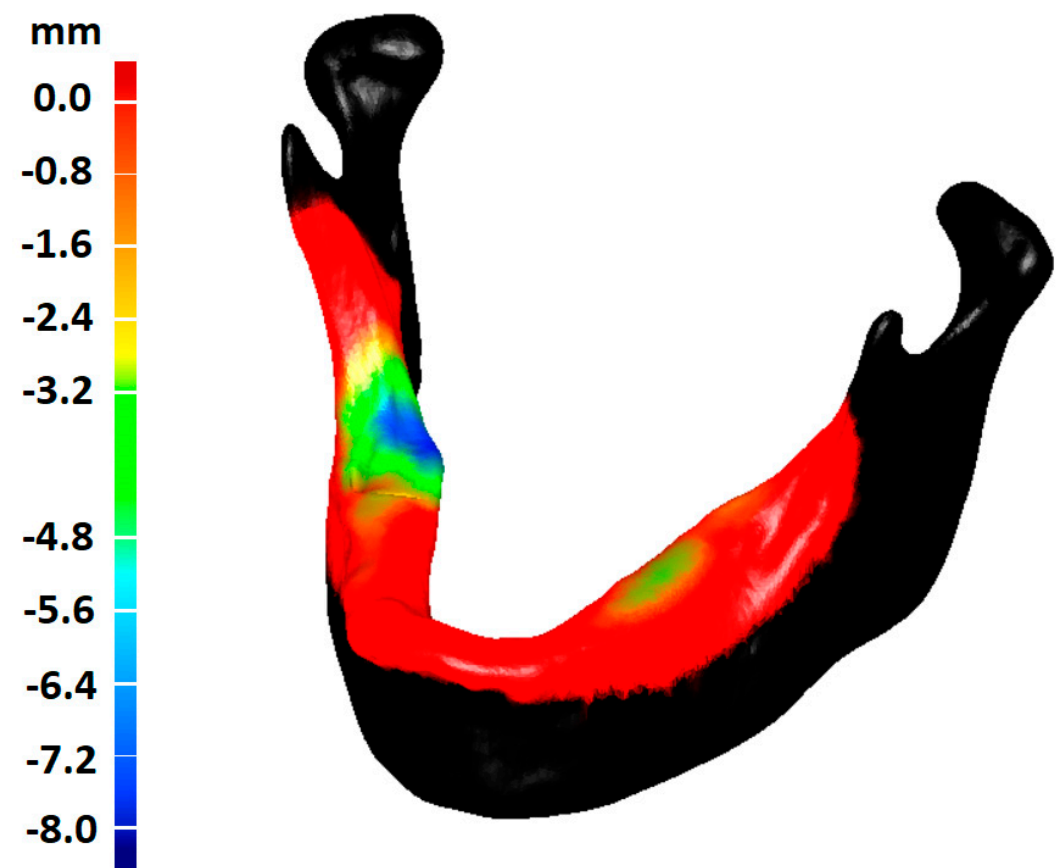

Figure 6. Indentation map representing the contact between maxilla and mandibula. 
Furthermore, on the left side (the healthy one) the load distribution was roughly uniform over the surface, while on the right side the distribution was nonhomogeneous. These results suggest that the exostosis can arise as a consequence of bone remodeling, resulting from a non-uniform distribution of loads on the right jawbone. According to Wolff's law [13], the bone reacts by remodeling itself, i.e., increases its size and resistant section, in response to the different intensities of the distributed loads on the mandibula. The different loads cause alterations in the structure of the bone substance and changes in the external conformation of the bones.

Applying the EFCM model and Equation (1), the intersection volumes on the right and left sides of the jawbones were extracted and calculated by Boolean operations, as shown in Figure 7. The intersection volume on the right side (where the exostosis arose) was $V_{R}=1797.92 \mathrm{~mm}^{3}$; the intersection volume on the left and healthy side was $\mathrm{V}_{\mathrm{L}}=346.44 \mathrm{~mm}^{3}$. According to these values, and to the offset value, the CM can be calculated on both sides according to the following:

$$
\mathrm{CM}_{\mathrm{R}}=\frac{\mathrm{V}_{\mathrm{R}}}{\Delta}=224.74 \mathrm{~mm}^{2} ; \mathrm{CM}_{\mathrm{L}}=\frac{\mathrm{V}_{\mathrm{L}}}{\Delta}=43.31 \mathrm{~mm}^{2} .
$$

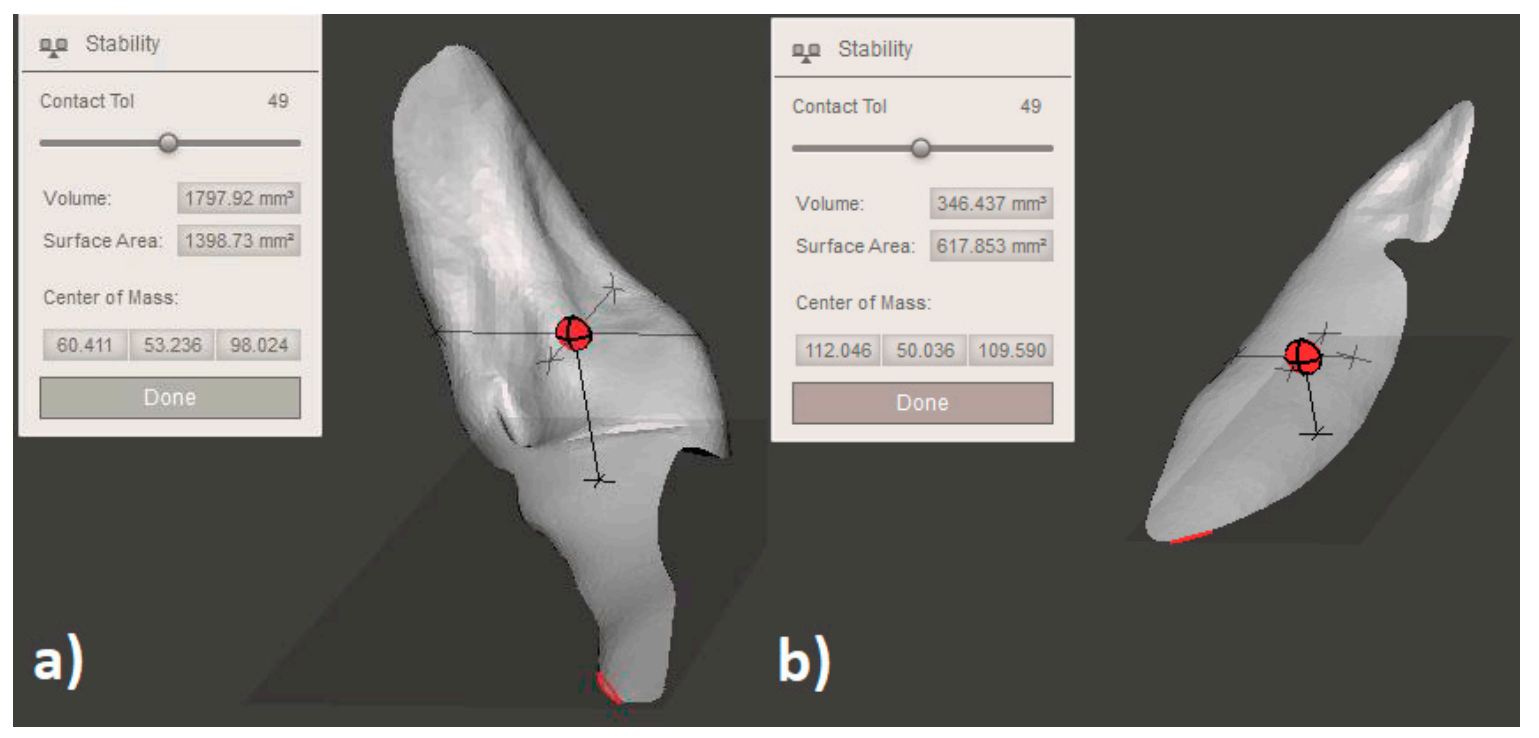

Figure 7. Intersection volumes for the congruence measure $(\mathrm{CM})$ calculation: right (a) and left (b) side of the jawbone.

In healthy mouths, the CM should have the same values on both contact sides of the jawbones. In this case study, the CM presented a higher value on the right side of the contact surfaces, where the exostosis grew to compensate the different load distributions on the two contact sides. This suggests that the comparison of the CM measures between maxilla and mandibula gives the opportunity to predict eventual bone growths.

\section{Conclusions}

This case study demonstrated how a maxillary exostosis correlated with mechanical contact disfunctions of the jawbones. The studied right buccal maxillary exostosis was analyzed by means of a 3D reconstruction from 2D CT images of the patient's cranium. The 3D model of the maxilla and mandibula was isolated, and geometrical calculations were obtained according to the joint congruence theory. The theory correlated the mechanical contact and the distribution of loads with geometrical indentations of the joint members. In this paper, the indentation map of the jawbone was reconstructed, and the distribution of loads was examined. Results show a non-uniform distribution of the loads between the right and left side of the jawbones, where the higher contact load was on the right side. 
This evidence, combined with the law of bone remodeling, points to the conclusion that exostosis can arise as the bone structure responds to contact disfunctions.

The purpose of this paper was to correlate the presence of exostoses with contact disfunction. This hypothesis was supported by the law of bone remodelling and the congruence calculation. In particular, the congruence calculation was done in order to give a mathematical consistency and explanation of the law of bone remodelling, which otherwise would be just a theory about how the bone reacts to loads and load disfunctions. In this way, the initial hypothesis could be demonstrated. Further in-depth analyses can be done in order to correlate the onset of an exostosis with other variables that influence bone remodeling, such as posture and patient's anamnesis. Furthermore, future work will be useful in confirming the findings of this paper by extending the same study to other case studies and different joints.

Author Contributions: Conceptualization, M.C.V.; data curation, S.L.; formal analysis, S.L.; methodology, M.C.V. and S.L.; project administration, M.C.V.; software, S.L.; supervision, M.C.V.; writing-original draft, M.C.V. and S.L.

Funding: This research received no external funding.

Conflicts of Interest: The authors declare no conflict of interest.

\section{References}

1. Sierra, A.C.; Jackson, I.T. A torus palatinus with a family history. Eur. J. Plast. Surg. 1992, 15, 98-99. [CrossRef]

2. Castellano, L.; Carini, F.; Minenna, L.; Lomartire, G.; Amosso, E. Exostoses of oral cavity: Classification and clinical aspects. Ital. Oral Surg. 2010, 9, 233-247. [CrossRef]

3. Regezi, J.A.; Sciubba, J.J. Oral Pathology: Clinico-Pathologic Correlations; WB Saunders Co.: Philadelphia, PA, USA, 1989; pp. 386-387.

4. Suzuki, M.; Sakai, T. A familial study of torus palatinus and torus mandibularis. Am. J. Phys. Anthropol. 1969, 18, 263-272. [CrossRef]

5. Reichart, P.A.; Neuhaus, F.; Sookasem, M. Prevalence of torus palatinus and torus mandibularis in Germans and Thais. Community Dent. Oral Epidemiol. 1988, 16, 61-64. [CrossRef] [PubMed]

6. Eggen, S. Torus mandibularis: An estimation of the degree of genetic determination. Acta Odontol. Scand. 1989, 47, 409-415. [CrossRef] [PubMed]

7. Gorsky, M.; Bukai, A.; Shohat, M. Genetic influence on the prevalence of torus palatinus. Am. J. Med. Genet. 1998, 75, 138-140. [CrossRef]

8. Bayer, I.S. Advances in Tribology of Lubricin and Lubricin-Like Synthetic Polymer Nanostructures. Lubricants 2018, 6, 30. [CrossRef]

9. Moss, M.L. The functional matrix hypothesis revisited. 1. The role of mechanotransduction. Am. J. Orthod. Dentofac. Orthop. 1997, 112, 8-11. [CrossRef]

10. Moss, M.L. The functional matrix hypothesis revisited. 2. The role of an osseous connected cellular network. Am. J. Orthod. Dentofac. Orthop. 1997, 112, 221-226. [CrossRef]

11. Moss, M.L. The functional matrix hypothesis revisited. 3. The genomic thesis. Am. J. Orthod. Dentofac. Orthop. 1997, 112, 338-342. [CrossRef]

12. Moss, M.L. The functional matrix hypothesis revisited. 4. The epigenetic antithesis and the resolving synthesis. Am. J. Orthod. Dentofac. Orthop. 1997, 112, 410-417. [CrossRef]

13. Wolff, J. The Law of Bone Remodelling; Springer: Berlin/Heidelberg, Germany, 1986.

14. Singh, G.D. On the etiology and significance of palatal and mandibular tori. Cranio J. Craniomandib. Pract. 2010, 28, 213-215. [CrossRef] [PubMed]

15. Pearson, O.M.; Lieberman, D.E. The Aging of Wolff's "Law": Ontogeny and Responses to Mechanical Loading in Cortical Bone. Yearb. Phys. Anthropol. 2004, 47, 63-99. [CrossRef] [PubMed]

16. Conconi, M.; Leardini, A.; Parenti-Castelli, V. Joint kinematics from functional adaptation: A validation on the tibio-talar articulation. J. Biomech. 2015, 48, 2960-2967. [CrossRef] [PubMed]

17. Conconi, M.; Parenti-Castelli, V. A sound and efficient measure of joint congruence. Proc. Inst. Mech. Eng. Part H J. Eng. Med. 2014, 228, 935-941. [CrossRef] [PubMed] 
18. Conconi, M.; Halilaj, E.; Parenti-Castelli, V.; Crisco, J.J. Is early osteoarthritis associated with differences in joint congruence? J. Biomech. 2014, 47, 3787-3793. [CrossRef] [PubMed]

19. Mazilu, T.; Gheti, M. On the vertical interaction between a three-axle bogie and track. In MATEC Web of Conferences; EDP Sciences: Les Ulis, France, 2017.

20. Li, D.; Smith, S.; Ma, X. End condition effect on initial buckling performance of thin plates resting on tensionless elastic or rigid foundations. Int. J. Mech. Sci. 2016, 105, 83-89. [CrossRef]

21. Voss, R.; Seeholzer, L.; Kuster, F.; Wegener, K. Analytical force model for orthogonal machining of unidirectional carbon fibre reinforced polymers (CFRP) as a function of the fibre orientation. J. Mater. Process. Technol. 2019, 263, 440-469. [CrossRef]

22. Valigi, M.C.; Papini, S. Analysis of chattering phenomenon in industrial S6-high rolling mill. Diagnostyka 2013, 14, 3-8.

23. Papini, S.; Valigi, M.C. Analysis of chattering phenomenon in industrial S6-high rolling mill. Part II: Experimental study. Diagnostyka 2016, 17, 27-32.

24. Valigi, M.C.; Cervo, S.; Petrucci, A. Chatter marks and vibration analysis in a S6-high cold rolling mill. Lect. Notes Mech. Eng. 2014, 5, 567-575.

25. Argatov, I.; Mishuris, G. Contact Mechanics of Articular Cartilage Layers: Asymptotic Models; Springer International Publishing: Cham, Switzerland, 2015.

(C) 2019 by the authors. Licensee MDPI, Basel, Switzerland. This article is an open access article distributed under the terms and conditions of the Creative Commons Attribution (CC BY) license (http:/ / creativecommons.org/licenses/by/4.0/). 